\title{
Preparation of CCISCRS Composite Based on WP Demoulding
}

\author{
Chuanbao $\mathrm{Wu}^{1, \mathrm{a}}$ \\ ${ }^{1}$ College of Chemistry and environmental engineering, Jiujiang University, Qianjin Eath Road \\ No.551, Jiujiang city, Jiangxi Province, People's Republic of China \\ awucbao@163.com
}

Keywords: Hot-pressing, Composite, Cellulose, Straw.

\begin{abstract}
In order to solve the question of sample damage encountered in the preparation of composite of carboxymethylcellulose (CC) and shortly cut rice straw (SCRS) by hot-pressing, waste paper (WP) was introduced as demoulding material. The tensile performance and hardness of composites at different CC mass contents were characterized. Results showed that WP had good demolding effect and integrate composite boards were easily obtained and sample damage was basically eliminated. Otherwise WP also showed certain strengthening role. The tensile strength increased by $88.8 \%$ at optimal conditions in comparison to the composites without WP. Tensile strength of the composite first increased and then decreased with increasing CC content. When CC content was $40 \%$, the composite reached its highest strength of $2.87 \mathrm{MPa}$. Tensile elongation showed similar changing law as tensile strength. The tensile elongation arrived at maximum of $9.36 \%$ when CC content was $40 \%$. Hardness of composites showed uneven property and maximum hardness waving range lay between 79 and 92 .
\end{abstract}

\section{Introduction}

Rice straw is a kind of renewable resource. Under the background of shortage of resources, developing rice straw based composite is of great significance. Otherwise, transformation of rice straw into composite is favorable to the decrease of pollution resulted from burning rice straw ${ }^{[1]}$. In the aspect of making environmentally friendly composites with rice straw as raw materials, there have been some reports ${ }^{[2-5]}$. When such composites were made, research focuses include the adhesive materials and preparation method. Rice straw is not only renewable resource but also degradable resource. If adhesive materials also have good degradable property, the composite prepared with such adhesive materials and rice straw has high environmental benefit and biological security.

In order to prepare completely degradable rice straw based composite, CC was selected as adhesive material and SCRS was selected as plant straw in our team. But the results were not desirable and the question was that composite had low strength and when samples were demolded, they were often easily damaged. As a way of solving this question, WP was introduced in the preparation of CC/SCRS composite.

\section{Materials and Methods}

Materials. Rice straw was obtained from Jiujiang suburbs farmland and was dried in the sun before use. It was cut into $1.5 \mathrm{~cm}$ about segments to obtain SCRS. CC was purchased from Hongda company (Heibei, China) and was used as received. WP was obtained from waste general exercise book. Water was from urban water-supplying system. Rapespeed oil was from common cooking oil and was used as auxiliary demolding agent.

Preparation of CC/SCRS Composite Based on WP Demoulding. Different amounts of SCRS were weighed into beaker and then $10 \mathrm{~mL}$ water was added, followed by stirring for 5 min so that SCRS was uniformly moistened. Thereafter, CC was weighed and added into moistened rice straw, followed by stirring for $5 \mathrm{~min}$. The total mass of SCRS and CC was kept as $30 \mathrm{~g}$. A $125 \mathrm{~mm} \times 125 \mathrm{~mm}$ $\times 2 \mathrm{~mm}$ iron mold was prepared by brushing a layer of rapespeed oil onto the top and bottom surfaces of mold and laying a sheet of WP on bottom surface. Then the mixed raw material was put in the 
prepared mold, another sheet of WP was laid on mixed raw material, upper mold plate was put on and then was hot-pressed on MZ-3012 machine at 10MPa pressure. After cooling to room temperature, the product was taken out to be tested.

Measurement of Composite. $2.5 \mathrm{~cm}$ wide strips were cut out from products to test tensile performance. Tensile strength and tensile elongation were measured on MZ-2000D.D1 electronic universal testing machine (Mingzhu test machine company, Jiangdu, Jiangsu, China) with tensile speed as $5 \mathrm{~mm} / \mathrm{min}$. Square with side length as $4 \mathrm{~cm}$ was selected from products and its four vertices were selected as hardness-measurement points. The four vertices were labeled respectively as point 1 , 2, 3 and 4 according to hardness-increasing order. Shore hardness was measured with LX-A Shore A type durometer (Mingzhu test machine company, Jiangdu, Jiangsu, China).

\section{Results and Discussion}

The Roles of WP and the Effect of CC Content on the Strength of SCRS/CC/WP Composite Materials. WP showed good demoulding effect and among study range of this paper all samples could be obtained easily. Damage of samples was eliminated because WP avoid the adhesion between composite and mold plate. Moreover WP adhered to samples after demoulding and became one part of composite. So the obtained composite was SCRS/CC/WP composite in fact. A series of SCRS/CC/WP composites were prepared at different mass contents of CC. The change of tensile strengths with the increase of CC content was drawn in Fig. 1. The mass content means the percentage ratio of the mass of CC to the total mass of CC and SCRS.

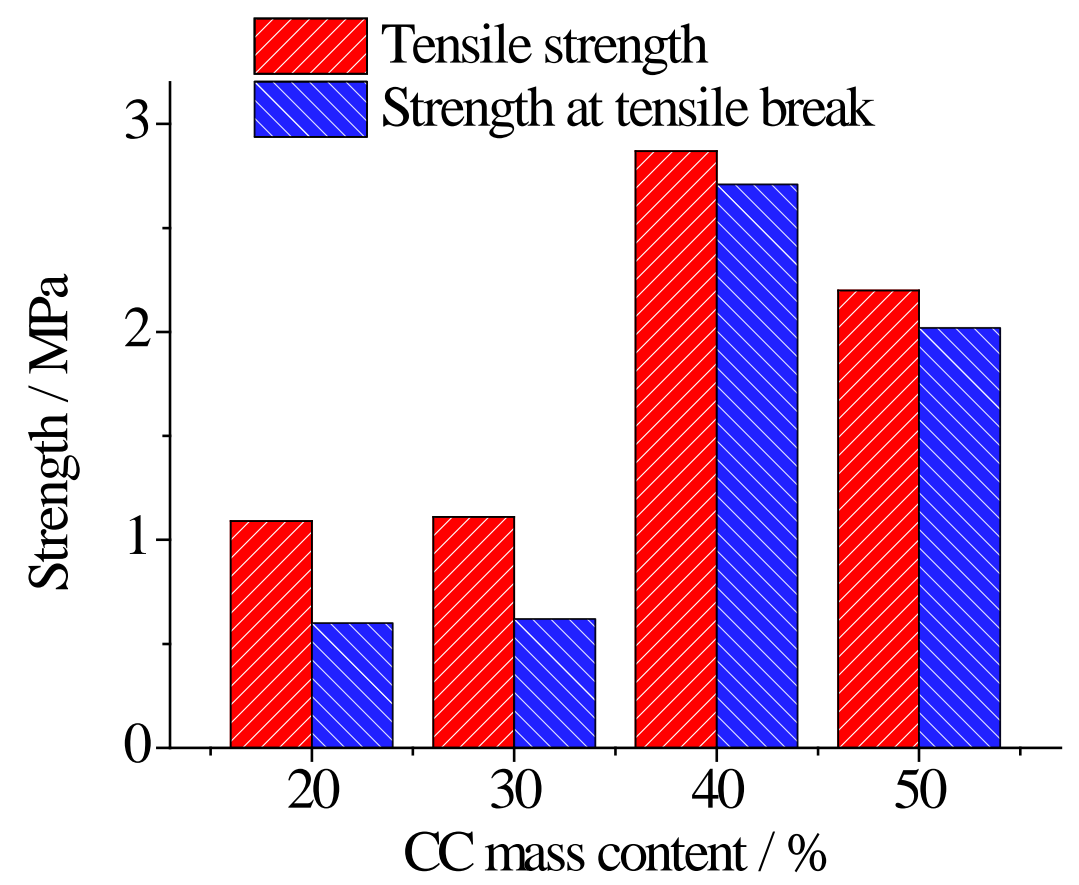

Fig. 1 The strength of composites at different CC contents.

It can be seen that the tensile strength of the composite first increases and then decreases with increasing CC content. When CC content is 40\%, the composite reaches its highest strength of 2.87 MPa. When WP was not used in our previous experiments, the best tensile strength was $1.52 \mathrm{MPa}$. Here the best value is $88.8 \%$ higher than previous best value. This indicates that WP bears not only demolding effect but also strengthening role. The strengthening role of WP comes from two aspects: one is its good inherent strength and the other is damage-avoiding of composite. In composites, CC acted as adhesive agent. When CC content was appropriately increased, more binding points resulted and so the strength of composites increased. But if CC was used too much, CC would aggregate and contain much inner structural defects, which resulted in the decrease of strength. Strength at tensile break also first increases and then decreases with increasing CC content. But the differences of these 
two strengths are different at all CC contents. Otherwise, it can be seen that tensile strength is higher than the strength at tensile break at different CC contents.

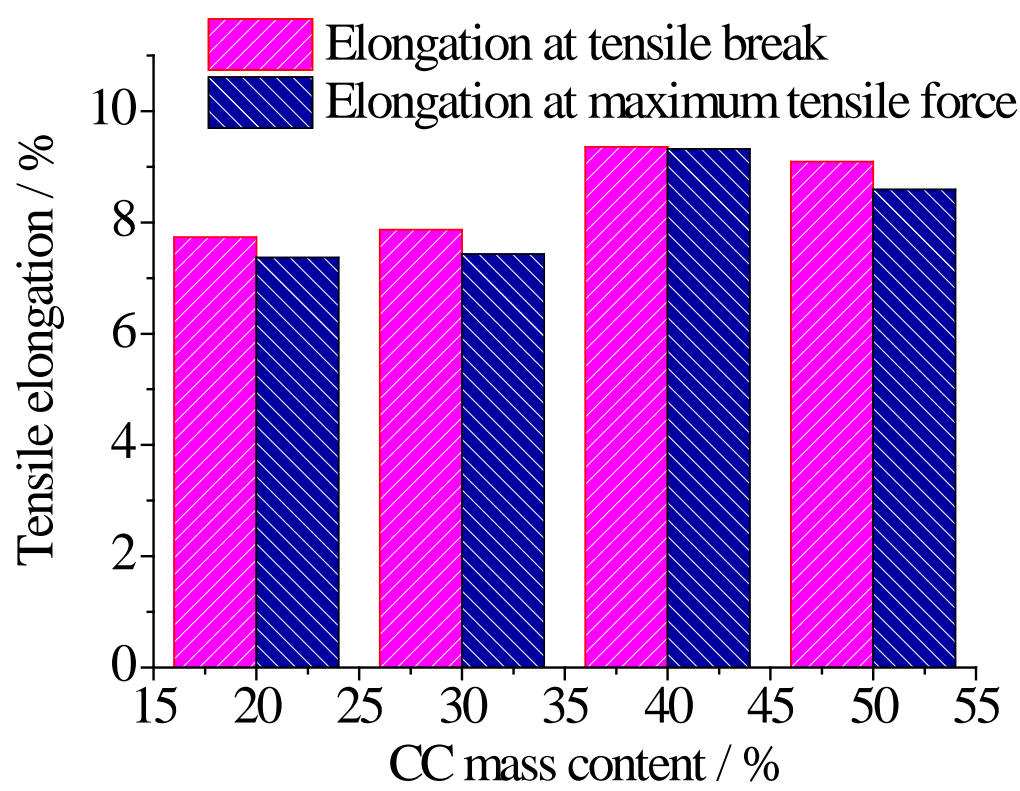

Fig. 2 The elongation of composites at different CC contents.

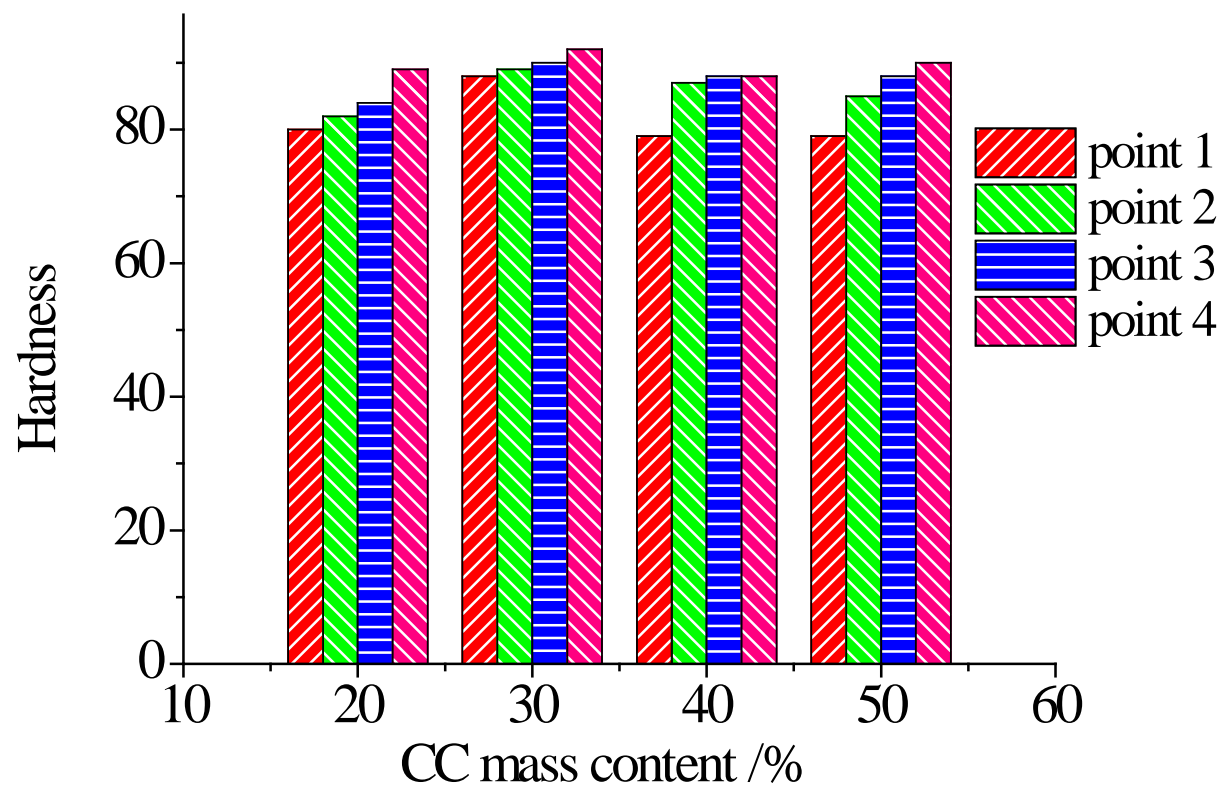

Fig. 3 The hardness of composites at different CC contents.

The Effect of CC Content on the Elongation of SCRS/CC/WP Composites. The changes of tensile elongation and elongation at maximum tensile force with increasing CC content were depicted in Fig 2. It can be seen that the two kinds of elongations of composite first increase and then decrease with increasing CC content and accord with change laws of strength. The tensile elongation arrives at maximum of $9.36 \%$ when CC content is $40 \%$. High strength means that more binding points without structural defects exist in composites and make composite have ability of withstanding longer drawing and so composites show high elongation value. It also can be seen that tensile elongations are generally higher than elongations at maximum tensile force except $40 \%$ mass content of CC. This indicates that SCRS/CC/WP composites have a little toughness. CC has very low toughness. So the 
toughness was thought to result from the native toughness of SCRS. In comparison to the composites prepared previously by our team, the elongation of SCRS/CC/WP composites was lower.

The Hardness of SCRS/CC/WP Composites. At different CC contents, the hardness of composites was depicted in Fig. 3. The hardness of composites shows two characteristics. First the hardness of even a sample is uneven. This indicates that the surface of composites has different flatness or composition. Second composites prepared at different CC mass contents have little difference of waving range of hardness. Maximum hardness waving range lies between 79 and 92 . Seen from the figure, hardness at $20 \%$ and 30\% CC content apparently was higher than that at $40 \%$ and 50\% CC content, which maybe resulted from normal random experimental error.

\section{Summary}

WP was very appropriate to prepare SCRS/CC composite materials. WP played two important roles. One was mold-releasing role and the other was strengthening role. The application of WP solved well the question of sample damage occurring in the direct preparation of SCRS/CC composite by hot-pressing. Whole samples were easily obtained among the range of CC mass content studied by this paper. WP has less structural defects than SCRS, so it can play a strengthening role. The best tensile strength of composite with WP is $88.8 \%$ higher than that of composite without WP. Broadening the application of WP is of certain significance and relevant work were being done by our team.

\section{Acknowledgements}

This work was financially supported by National Natural Science Foundation of China (21164002).

\section{References}

[1] N. Said, T. Bishara, A. García-Maraver and M. Zamorano: Waste Manage. Vol. 33(2013), p. 2250

[2] F. Gu, W.X. Wang, L. Jing and Y.C. Jin: Bioresource Technol. Vol. 149(2013), p. 375

[3] C.Y. Mu, M. Jiang, J. Zhu, M.M. Zhao, S.B Zhu and Z.W. Zhou: Renew. Energ. Vol. 63(2014), p. 324

[4] K. Suresh, A. Ranjan, S. Singh and V.S. Moholkar: Ultrason. Sonochem. Vol. 21(2014), p. 200

[5] J.J. Liu, C.J. Jia and C.X. He: AASRI Procedia Vol. 3(2012), p. 83

[6] F. Yao, Q.L. Wu, Y. Lei and Y.J. Xu: Ind. Crop. Prod. Vol. 28 (2008), p. 63

[7] Y. Zhao, J.H. Qiu, H.X. Feng, M. Zhang, L. Lei, X.L. Wu: Chem. Eng. J. Vol. 173(2011), p. 659

[8] L.J. Qin, J.H. Qiu, M.Z. Liu, S.L. Ding, L. Shao, S.Y. Lü, G.H. Zhang, Y. Zhao, X. Fu: Chem. Eng. J. Vol. 166(2011), p. 772 